\title{
Unexpected enhancements and reductions of rf spin resonance strengths
}

\author{
M. A. Leonova, V. S. Morozov, A. D. Krisch, R. S. Raymond, D. W. Sivers, and V. K. Wong \\ Spin Physics Center, University of Michigan, Ann Arbor, Michigan 48109-1120, USA \\ R. Gebel, A. Lehrach, B. Lorentz, R. Maier, D. Prasuhn, A. Schnase, and H. Stockhorst \\ Forschungszentrum Jülich, Institut für Kernphysik, Postfach 1913, D-52425 Jülich, Germany \\ F. Hinterberger and K. Ulbrich \\ Helmholtz-Institut für Strahlen- und Kernphysik, Universität Bonn, D-53115 Bonn, Germany
}

(Received 31 March 2006; published 8 May 2006)

\begin{abstract}
We recently analyzed all available data on spin-flipping stored beams of polarized protons, electrons, and deuterons. Fitting the modified Froissart-Stora equation to the measured polarization data after crossing an rf-induced spin resonance, we found 10-20-fold deviations from the depolarizing resonance strength equations used for many years. The polarization was typically manipulated by linearly sweeping the frequency of an rf dipole or rf solenoid through an rf-induced spin resonance; spin-flip efficiencies of up to $99.9 \%$ were obtained. The Lorentz invariance of an rf dipole's transverse $\int B d l$ and the weak energy dependence of its spin resonance strength $\mathcal{E}$ together imply that even a small rf dipole should allow efficient spin flipping in $100 \mathrm{GeV}$ or even $\mathrm{TeV}$ storage rings; thus, it is important to understand these large deviations. Therefore, we recently studied the resonance strength deviations experimentally by varying the size and vertical betatron tune of a $2.1 \mathrm{GeV} / c$ polarized proton beam stored in COSY. We found no dependence of $\mathcal{E}$ on beam size, but we did find almost 100-fold enhancements when the rf spin resonance was near an intrinsic spin resonance.
\end{abstract}

DOI: 10.1103/PhysRevSTAB.9.051001

PACS numbers: 29.27.Bd, 29.27.Hj, 41.75.Ak

\section{INTRODUCTION}

Polarized beam experiments have become an important part of the programs in storage rings such as the IUCF Cooler Ring [1], AmPS at NIKHEF [2], the MIT-Bates Storage Ring [3], COSY [4], LEP at CERN [5], RHIC at BNL [6] and HERA at DESY [7,8]. To reduce their systematic errors, polarized scattering experiments require frequent spin-direction reversals (spin flips) while the polarized beam is stored. Spin resonances [9-11] induced by either an rf solenoid or rf dipole can produce these spin flips in a well-controlled way [12-25].

In any flat storage ring or circular accelerator with no horizontal magnetic fields, each beam particle's spin precesses around the vertical fields of the ring's dipole magnets. The spin tune $\nu_{s}$, which is the number of spin precessions during one turn around the ring, is proportional to the particle's energy

$$
\nu_{s}=G \gamma,
$$

where $G=(g-2) / 2$ is the particle's gyromagnetic anomaly $\left(G_{p}=1.792847, G_{e}=1.15967 \times 10^{-3}\right.$, and $\left.G_{d}=-0.142987\right)$ and $\gamma$ is its Lorentz energy factor. The vertical polarization can be perturbed by an rf magnet's horizontal rf magnetic field. This perturbation can induce an rf depolarizing resonance, which can flip the spin direction of stored polarized particles [12-25]; the reso- nance's frequency is

$$
f_{r}=f_{c}\left(k \pm \nu_{s}\right),
$$

where $f_{c}$ is the particle's circulation frequency and $k$ is an integer.

Ramping an rf magnet's frequency through $f_{r}$ can flip each particle's spin. The modified [15,19] Froissart-Stora equation [9] relates the beam's initial polarization $P_{i}$ to its final polarization $P_{f}$ after crossing the resonance,

$$
P_{f}=P_{i}\left\{(1+\hat{\eta}) \exp \left[\frac{-\left(\pi \mathcal{E}_{F S} f_{c}\right)^{2}}{\Delta f / \Delta t}\right]-\hat{\eta}\right\} ;
$$

the parameter $\hat{\eta}$ is the limiting spin-flip efficiency and the ratio $\Delta f / \Delta t$ is the resonance crossing rate, where $\Delta f$ is the ramp's frequency range during the ramp time $\Delta t$. Equation (3) is valid if $\Delta f$ is larger than the spin resonance's width. We will later discuss how one obtains the spin resonance strength $\mathcal{E}_{F S}$ from Eq. (3).

One can also obtain the resonance strength $\mathcal{E}_{B d l}$ due to an $\mathrm{rf}$ solenoid or rf dipole using

$$
\begin{aligned}
& \text { solenoid: } \quad \mathcal{E}_{B d l}=\frac{1}{\pi \sqrt{2}} \frac{e(1+G)}{p} \int B_{\mathrm{rms}} d l, \\
& \text { dipole : } \quad \mathcal{E}_{B d l}=\frac{1}{\pi \sqrt{2}} \frac{e(1+G \gamma)}{p} \int B_{\mathrm{rms}} d l,
\end{aligned}
$$


where $e$ is the particle's charge, $p$ is its momentum, and $\int B_{\mathrm{rms}} d l$ is the rf magnet's rms magnetic field integral in its rest frame [26-28]. These equations are for an ideal flat circular accelerator with a point rf magnet causing the only perturbation of the spin motion. Other authors [29,30] derived a similar equation that differed by a factor of 2 in the denominator

$$
\text { dipole : } \quad \mathcal{E}_{B d l}=\frac{1}{\pi 2 \sqrt{2}} \frac{e(1+G \gamma)}{p} \int B_{\mathrm{rms}} d l .
$$

\section{ANALYSIS OF EXISTING DATA}

To understand this factor of 2 difference, we made a compilation of all available experimental data [31] that allowed a simultaneous evaluation of the spin resonance strength $\mathcal{E}_{B d l}$, obtained from Eq. (4) or (5), and the spin resonance strength $\mathcal{E}_{F S}$ obtained from Eq. (3), the modified Froissart-Stora formula. This compilation indicated that for many experiments $\mathcal{E}_{B d l}$ and $\mathcal{E}_{F S}$ disagree with both $[26,27]$ and $[29,30]$ by factors of $0.1,10$, or more.

The resonance strength $\mathcal{E}_{F S}$ can be obtained by first measuring the final beam polarization $P_{f}$ after ramping an rf magnet's frequency by a range $\Delta f$ during a time $\Delta t$ through a spin resonance; then $P_{f}$ is plotted vs the different $\Delta t$ values and the data are fit to Eq. (3) with $\mathcal{E}_{F S}$ as a fit parameter. Examples of these curves are shown in Figs. 1 and 2 for protons [23] and deuterons [25], respectively. For protons, using Eq. (5) gave $\mathcal{E}_{B d l}$ of $(80 \pm 4) \times 10^{-6}$, while $\mathcal{E}_{F S}$ was $(580 \pm 10) \times 10^{-6}$, which is about 7.3 times larger. For deuterons, $\mathcal{E}_{B d l}$ was $(16 \pm 1) \times 10^{-6}$, while

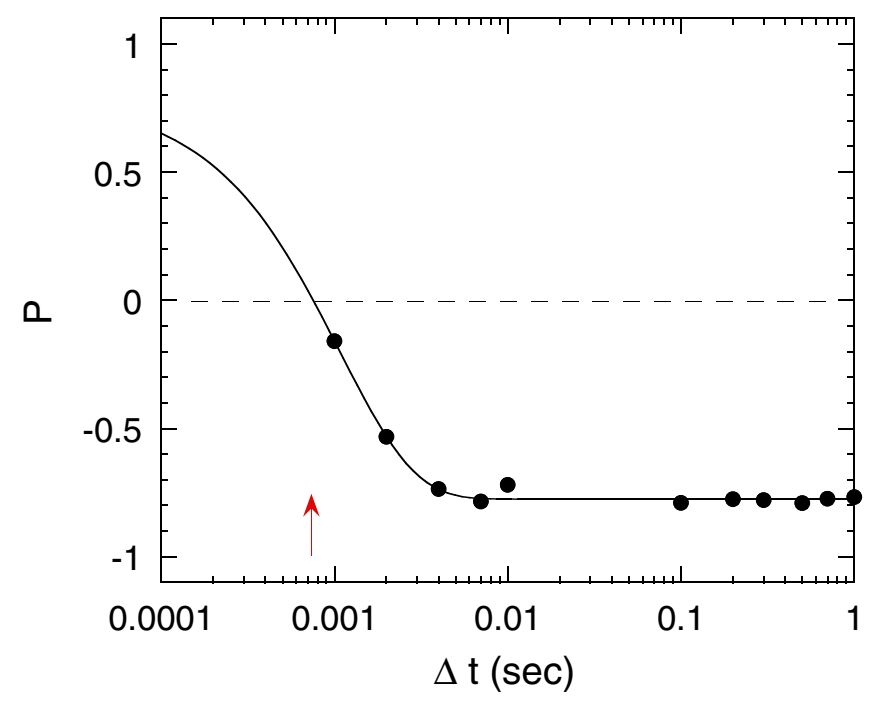

FIG. 1. (Color) Measured proton polarization at $2.1 \mathrm{GeV} / c$ is plotted vs rf dipole ramp time $\Delta t$ [23]. The rf dipole's frequency range $\Delta f$ was $8 \mathrm{kHz}$; its $\int B d l$ was $0.46 \pm 0.03 \mathrm{~T} \mathrm{~mm}$; thus, Eq. (5) gives $\mathcal{E}_{\mathrm{Bdl}}=(80 \pm 4) \times 10^{-6}$. The fit to Eq. (3) gives $\mathcal{E}_{\mathrm{FS}}=(580 \pm 10) \times 10^{-6}$; the arrow shows the $\Delta t$ where $P=0$.

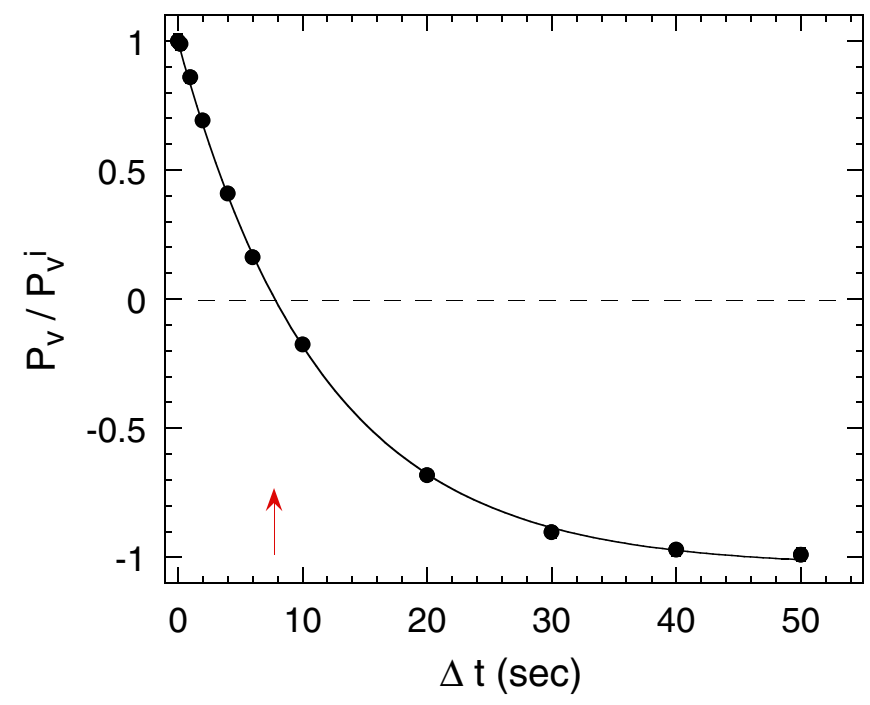

FIG. 2. (Color) Measured average vector deuteron polarization ratios at $1850 \mathrm{MeV} / c$ are plotted vs rf dipole ramp time $\Delta t$ [25]. The rf dipole's frequency range $\Delta f$ was $200 \mathrm{~Hz}$; its $\int B d l$ was $0.54 \pm 0.03 \mathrm{~T} \mathrm{~mm}$; thus, Eq. (5) gives $\mathcal{E}_{\mathrm{Bdl}}=(16 \pm 1) \times 10^{-6}$. The fit to Eq. (3) gives $\mathcal{E}_{\mathrm{FS}}=(1.17 \pm 0.01) \times 10^{-6}$; the arrow shows the $\Delta t$ where $P=0$.

$\mathcal{E}_{F S}$ was $(1.17 \pm 0.01) \times 10^{-6}$, which is about 14 times smaller. Note that these two experiments were both done with the same rf dipole at COSY; thus, these large strength deviations could not be due to incorrect calibrations of $\int B d l$.

We examined all available publications and logbooks on spin-flipping experiments [31] that had both: information on the rf magnet's $\int B d l$, and data for a polarization vs ramp time $\Delta t$ curve. We also checked in each experiment for any other spin resonances near the rf-induced resonance. Fits to Eq. (3), the modified Froissart-Stora equation, gave the measured spin resonance strength for each experiment and its error. We also calculated the spin resonance strength from each rf magnet's $\int B d l$ using Eq. (4) or (5). For the experiments at IUCF and MIT, there were no precise measurements of the rf magnet's $\int B d l$; thus, we assumed a $\pm 10 \%$ error. For the more recent experiments at COSY, the rf magnet's $\int B d l$ was known with a $\pm 5 \%$ uncertainty.

Then we took the ratio of the two spin resonance strengths, $\mathcal{E}_{F S} / \mathcal{E}_{B d l}$, for each experiment and obtained its error using simple error propagation formulas. Figure 3 shows these ratios plotted vs the ramp time $\Delta t$ at which $P$ passes through 0 in each $\Delta t$ curve. The dashed [26-28] and solid $[29,30]$ lines show the predicted ratios.

\section{COSY EXPERIMENT}

To better understand these deviations, we recently studied experimentally the dependence of an rf resonance's strength on the beam size and on its distance from an 

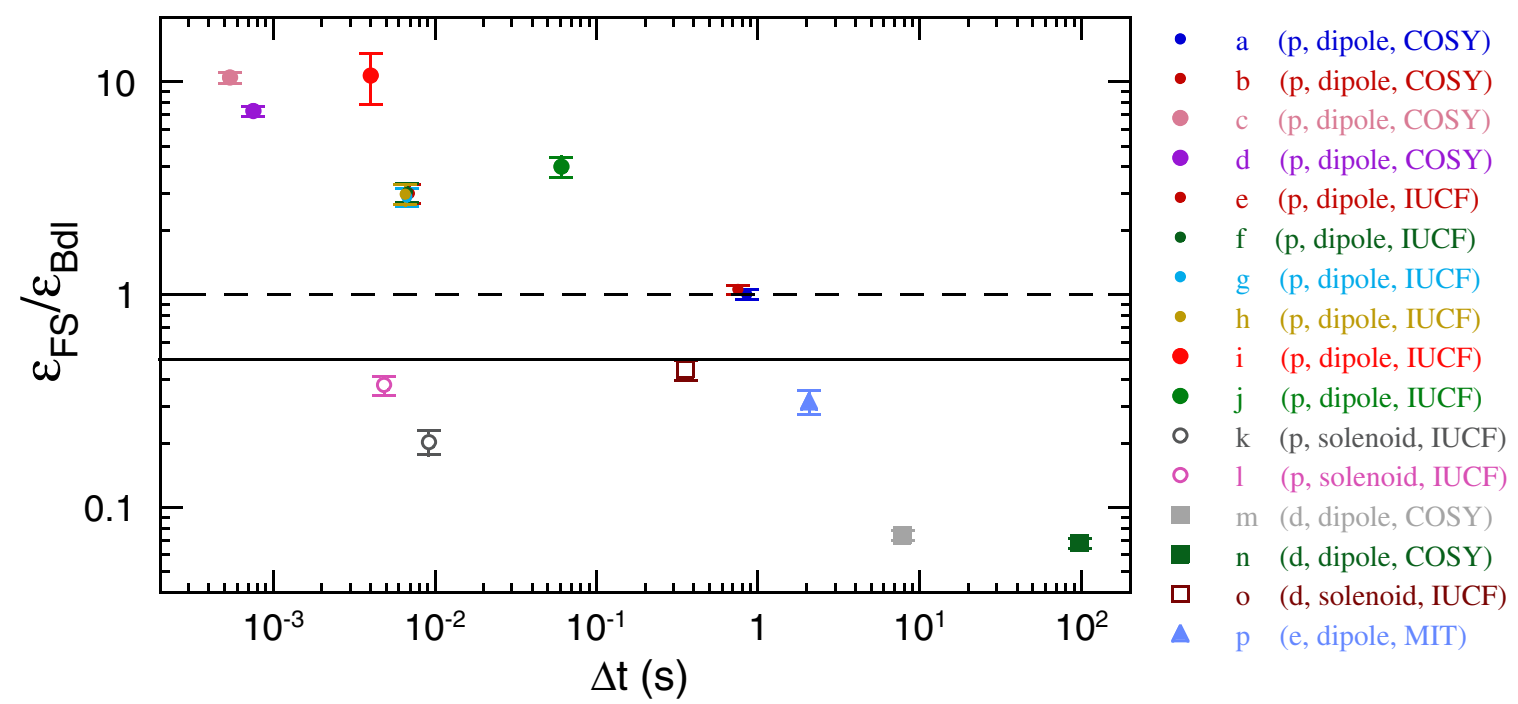

FIG. 3. (Color) Ratio of $\mathcal{E}_{F S}$ to $\mathcal{E}_{B d l}$ is plotted vs the ramp time $\Delta t$ giving $P=0$ in each $\Delta t$ curve [31]; for example, points (d) and (m) come from Figs. 1 and 2, respectively. $\mathcal{E}_{F S}$ is the resonance strength obtained by fitting data in each $\Delta t$ curve to Eq. (3), the modified Froissart-Stora equation; $\mathcal{E}_{B d l}$ is obtained using each data point's $\int B d l$ in Eq. (4) or (5). The dashed and solid lines show the ratios predicted by [26-28] and [29,30], respectively. Note that points (a) and (b) overlap, as do points (e)-(h). The data for all points are listed in Table I.

intrinsic spin resonance. We did this by varying the size and the vertical betatron tune of a $2.1 \mathrm{GeV} / c$ polarized proton beam stored in COSY.

The apparatus used for this experiment, including the COSY storage ring [32-35], the EDDA detector [36], the electron Cooler [37], the low energy polarimeter, the injector cyclotron, and the polarized ion source [38-40], are indicated in Fig. 4, along with the rf dipole. The beam emerging from the polarized $\mathrm{H}^{-}$ion source was accelerated by the cyclotron to COSY's $45 \mathrm{MeV}$ injection energy. Then the low energy polarimeter measured the beam's polarization before injection into COSY to monitor the stable operation and polarization of the ion source. The electron Cooler reduced the beam's size and momentum spread at injection energy. A $24.5 \mathrm{keV}$ electron beam of about $170 \mathrm{~mA}$, with a diameter of about $25 \mathrm{~mm}$, cooled the protons in a 2-m-long cooling section; it took $10 \mathrm{~s}$ to cool the proton beam to its equilibrium emittances in both the longitudinal and transverse dimensions. This decreased its initial momentum spread by about a factor of 10 to $\Delta p / p=9 \times 10^{-5}$ FWHM.

We manipulated the beam's polarization using a ferritecore rf dipole, which contained a copper coil of 8 turns; their spacing was optimized to produce a uniform radial magnetic field. The $\mathrm{rf}$ dipole was part of an LC resonant circuit, which operated near $f_{r}=902.6 \mathrm{kHz}$, at an $\mathrm{rf}$ voltage of $1 \mathrm{kV}$ rms giving an $\mathrm{rf} \int B_{\mathrm{rms}} d l$ of $0.19 \pm 0.01 \mathrm{Tmm}$.

We measured the polarization in COSY using the EDDA detector $[4,36]$; we reduced its systematic errors by cycling the polarized source between the up and down vertical

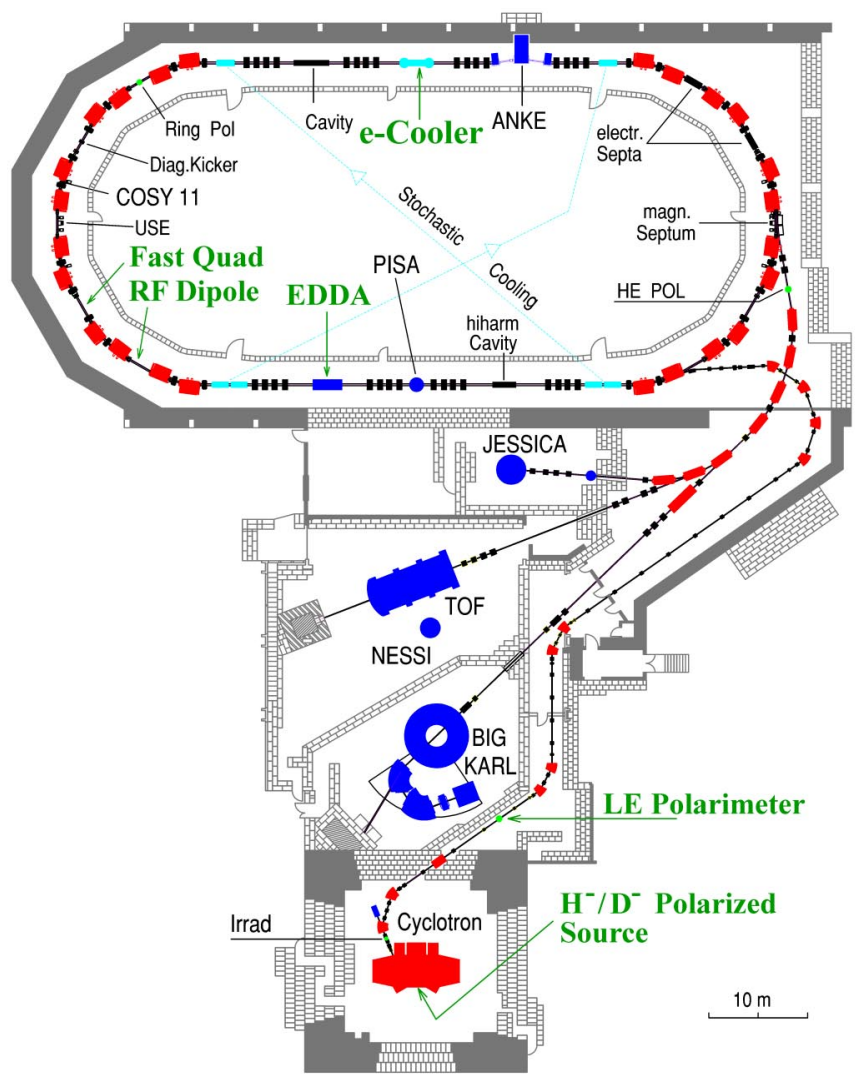

FIG. 4. (Color) Layout of the COSY storage ring, with its injector cyclotron and polarized ion source. Also note the $\mathrm{rf}$ dipole, the fast quadrupole, EDDA, the low energy (LE) polarimeter, and the electron Cooler. 


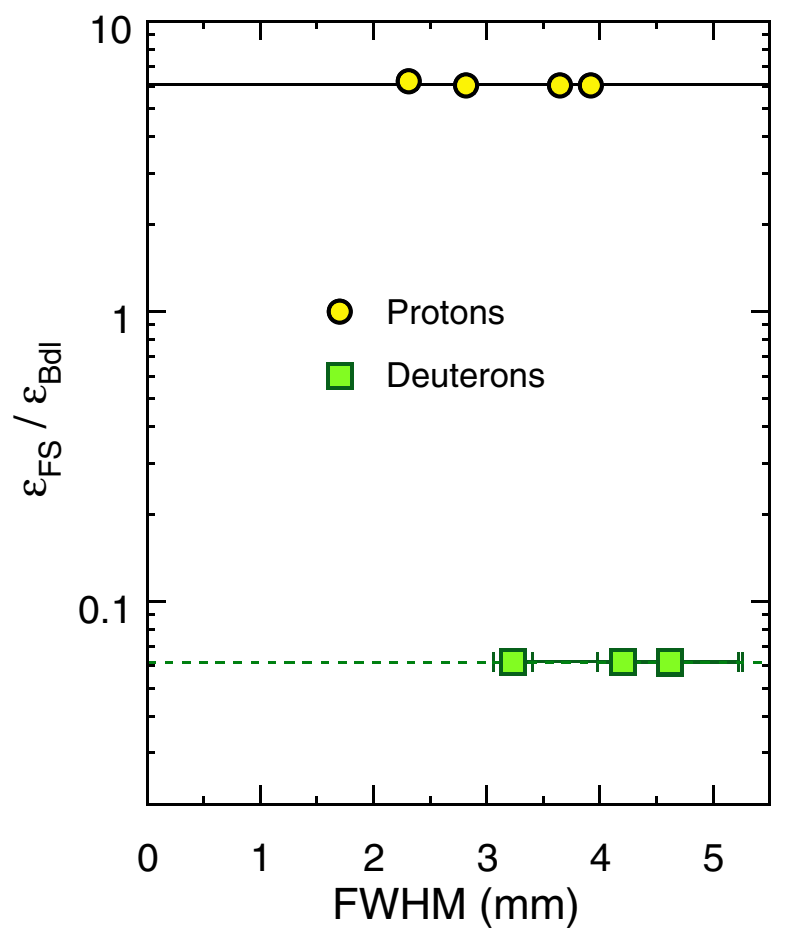

FIG. 5. (Color) Ratio of $\mathcal{E}_{F S}$ to $\mathcal{E}_{B d l}$ is plotted vs the vertical beam size (FWHM). $\mathcal{E}_{F S}$ is the resonance strength obtained by fitting the $\Delta t$ curve for each data point to Eq. (3); $\mathcal{E}_{B d l}$ is obtained using each data point's $\int B d l$ in Eq. (5). Fits to horizontal straight lines give resonance strength ratios of $6.07 \pm 0.04$ for protons and $0.0618 \pm 0.0002$ for deuterons. The $\nu_{y}$ values were 3.525 for protons and 3.60 for deuterons. polarization states. The $\mathrm{rf}$ acceleration cavity was turned off and shorted during COSY's flattop; thus, there were no synchrotron sideband effects $[14,41,42]$. The measured flattop polarization, before spin manipulation, was typically $50 \%$ to $60 \%$.

We determined the resonance's position and the upper limit on its width using the procedure described in [23]. Then, we obtained the resonance strength $\mathcal{E}_{F S}$, as in Figs. 1 and 2 , for different fast quadrupole strengths. The fast quadrupole increased the beam's emittance at the start of COSY's flattop, before we manipulated the beam's polarization with the rf dipole. We measured beam's vertical profile for each fast quadrupole strength by moving a thin fiber target vertically through the beam, while measuring the current due to its scattering events as the fiber moved. The $\mathcal{E}_{F S} / \mathcal{E}_{B d l}$ ratios are plotted against the beam's vertical size (FWHM) in Fig. 5. We also plot deuteron data obtained in a similar way during a December 2004 run [25]. The proton and deuteron data both show no dependence on the beam's vertical size. The fits to horizontal straight lines give resonance strength ratios of $6.07 \pm 0.04$ for protons and $0.0618 \pm 0.0002$ for deuterons.

We also obtained the resonance strength $\mathcal{E}_{F S}$, as in Figs. 1 and 2, for different values of the vertical betatron tune $\nu_{y}$. We plotted the resulting $\mathcal{E}_{F S} / \mathcal{E}_{B d l}$ ratios against $\nu_{y}$ in Fig. 6; we also plotted our 2004 deuteron data [25]. Notice the $\nu_{s}=8-\nu_{y}$ first-order intrinsic spin resonance for protons, which let us study the rf resonance strength in its proximity; there were also four third-order resonances.

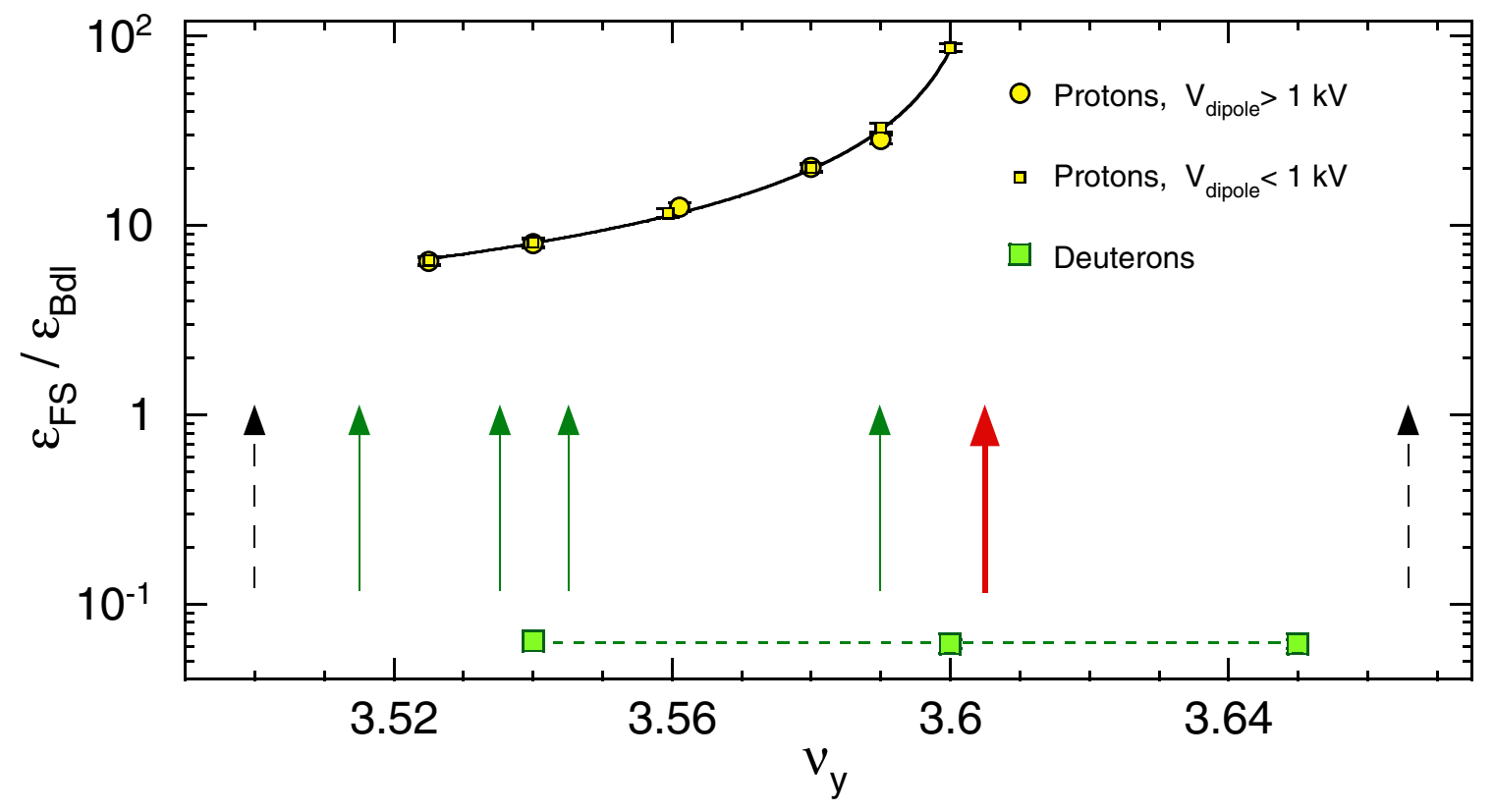

FIG. 6. (Color) Ratio of $\mathcal{E}_{F S}$ to $\mathcal{E}_{B d l}$ is plotted vs the vertical betatron tune $\nu_{y}$. $\mathcal{E}_{F S}$ is the resonance strength obtained by fitting the data in the $\Delta t$ curve for each $\nu_{y}$ to Eq. (3); $\mathcal{E}_{B d l}$ is obtained using each data point's $\int B d l$ in Eq. (5). The proton data fit to Eq. (7), gives $\nu_{r}$ of $3.6060 \pm 0.0005, A$ of $0.44 \pm 0.46$ and $B$ of $0.50 \pm 0.03$. Fitting the deuteron data to a horizontal straight line gives a resonance strength ratio $\mathcal{E}_{F S} / \mathcal{E}_{B d l}=0.063 \pm 0.002$. The betatron beam resonances are shown by the black dashed arrows; the 1st and 3rd order proton spin resonances are shown by the red (bold) and green arrows, respectively. 
Using references [43,44], one can obtain the dependence of $\mathcal{E}_{F S} / \mathcal{E}_{B d l}$ on the distance between the vertical betatron tune $\nu_{y}$ and the rf spin resonance's tune $\nu_{r} \equiv k \pm f_{r} / f_{c}$, where $k$ is an integer; this dependence is

$$
\mathcal{E}_{F S} / \mathcal{E}_{B d l}=A+\frac{B}{\left|\nu_{y}-\nu_{r}\right|} .
$$

Fitting the proton data in Fig. 6 to Eq. (7) gave $A$ of $0.44 \pm 0.46, \quad B$ of $0.50 \pm 0.03, \quad$ and $\nu_{r}$ of
$3.6060 \pm 0.0005$, which was very near the calculated value of 3.605 for the first-order intrinsic spin resonance. The parameter $B$ depends on many details of the ring. The parameter $A$ should give the predicted resonance strength ratio $\mathcal{E}_{F S} / \mathcal{E}_{B d l}$ of either 1 [26-28] or $0.5[29,30]$ when one is infinitely far from any intrinsic spin resonances. Clearly our data could not determine $A$ with good precision.

The deuteron data in Fig. 6 show no dependence on $\nu_{y}$; its fit to a horizontal straight line gave a resonance strength ratio of $0.063 \pm 0.002$, which agrees with the result from
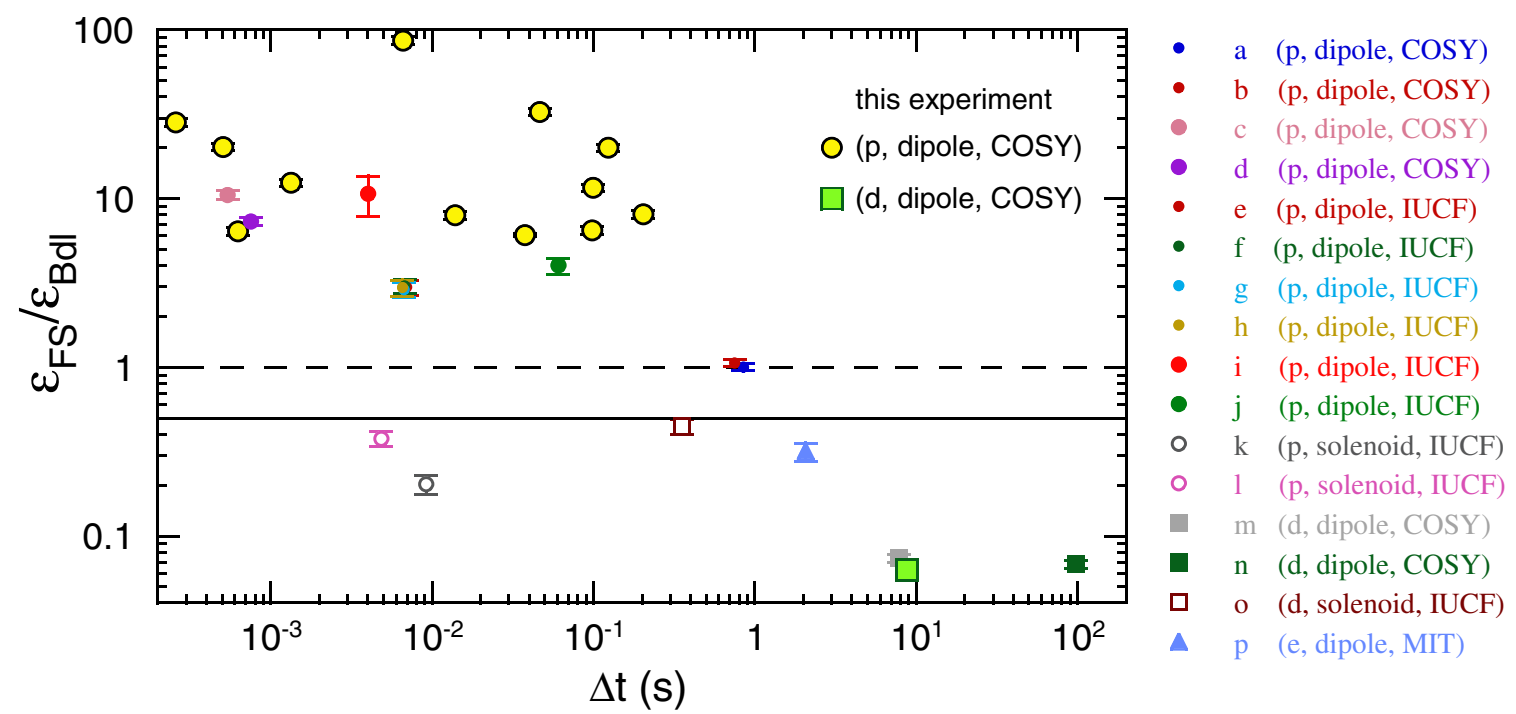

FIG. 7. (Color) Ratio of $\mathcal{E}_{F S}$ to $\mathcal{E}_{B d l}$ is plotted vs the ramp time $\Delta t$ giving $P=0$ in each $\Delta t$ curve [31]. $\mathcal{E}_{F S}$ is the resonance strength obtained by fitting the data in each $\Delta t$ curve to Eq. (3); $\mathcal{E}_{B d l}$ is obtained using each data point's $\int B d l$ in Eq. (4) or (5). The dashed and solid lines show the ratios predicted by [26-28] and [29,30], respectively. Note that points (a) and (b) overlap, as do points (e)-(h). The data for all points are listed in Table I.
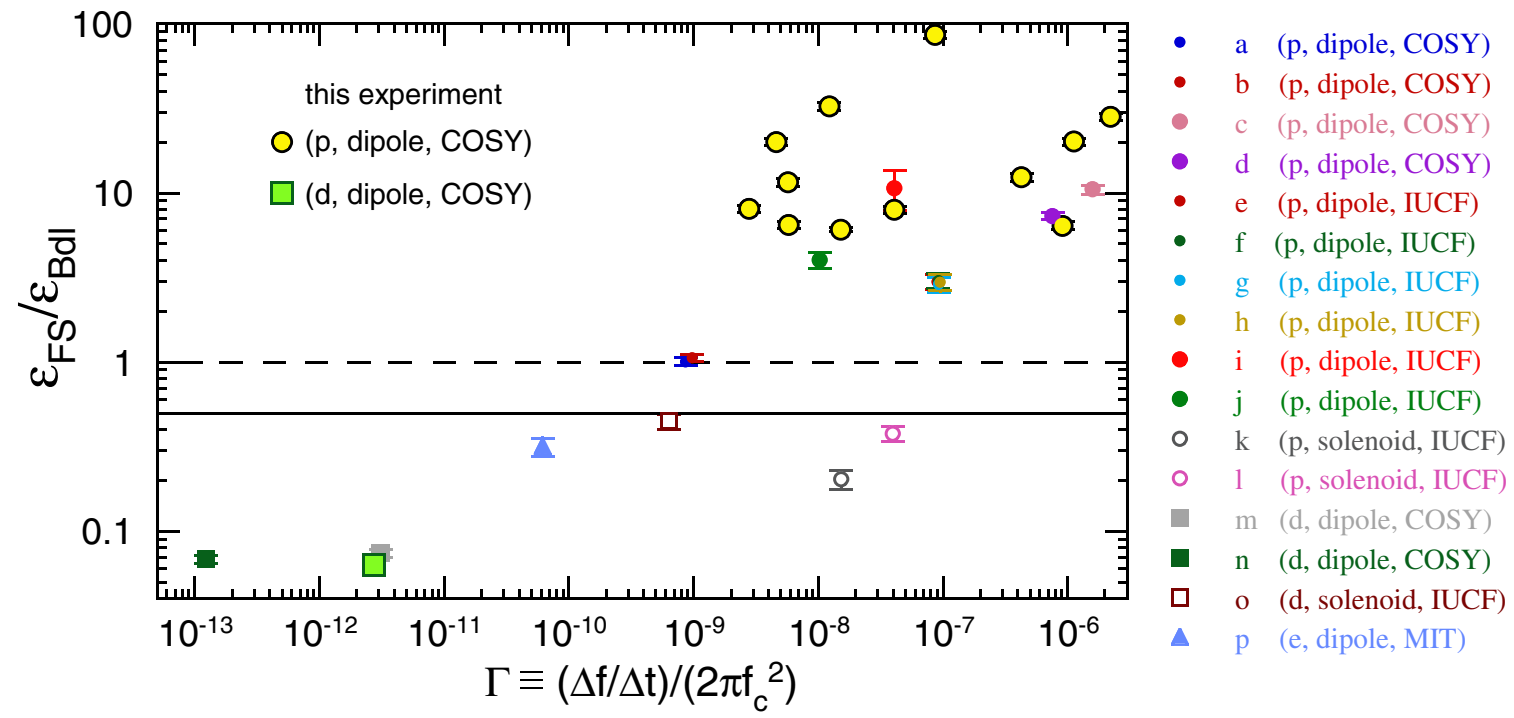

FIG. 8. (Color) Ratio of $\mathcal{E}_{F S}$ to $\mathcal{E}_{B d l}$ is plotted vs the dimensionless crossing rate $\Gamma$ giving $P=0$ in each $\Delta t$ curve [31]. $\mathcal{E}_{F S}$ is the resonance strength obtained by fitting the data in each $\Delta t$ curve to Eq. (3); $\mathcal{E}_{B d l}$ is obtained using each data point's $\int B d l$ in Eq. (4) or (5). The dashed and solid lines show the ratios predicted by [26-28] and [29,30], respectively. Note that points (a) and (b) overlap, as do points (e)-(h). The data for all points are listed in Table I. 


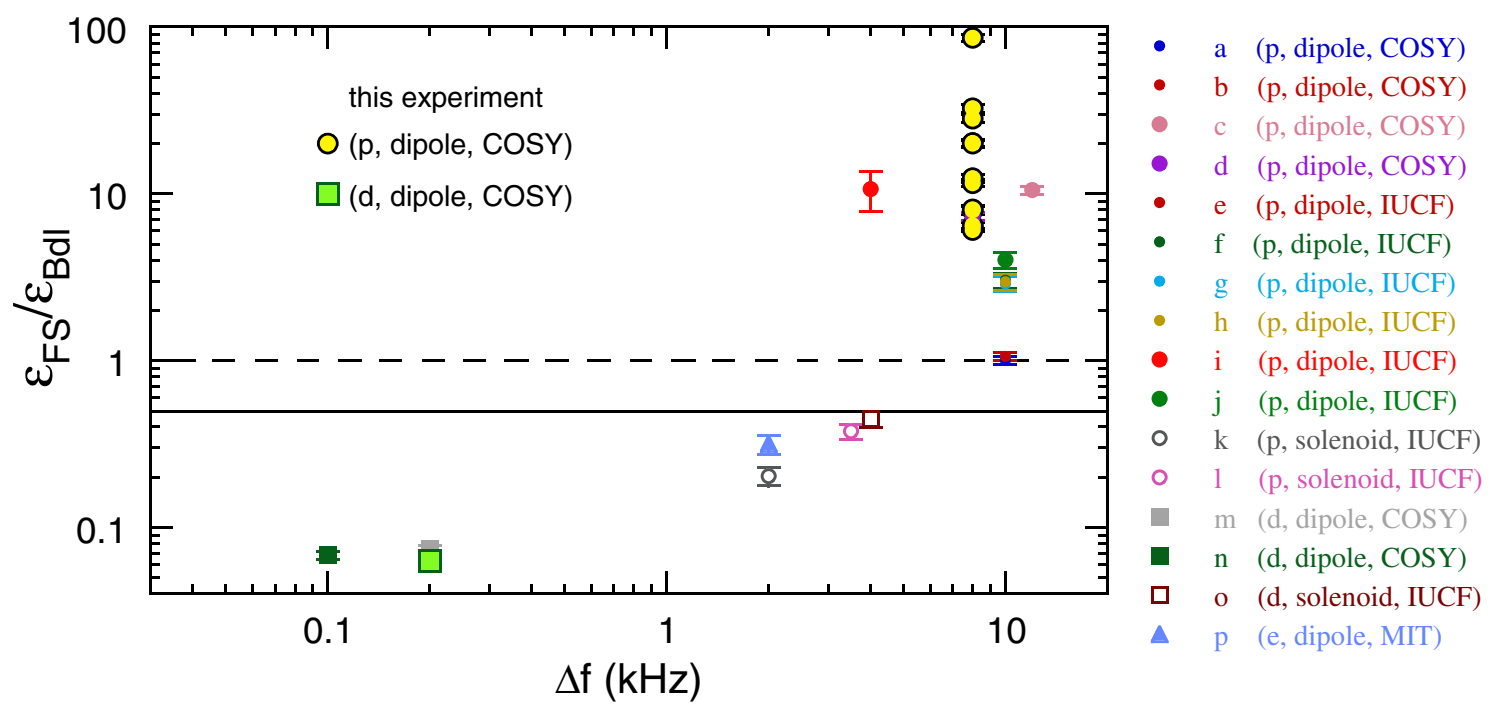

FIG. 9. (Color) Ratio of $\mathcal{E}_{F S}$ to $\mathcal{E}_{B d l}$ is plotted vs the frequency ramp range $\Delta f$ used for each $\Delta t$ curve [31]. $\mathcal{E}_{F S}$ is the resonance strength obtained by fitting the data in each $\Delta t$ curve to Eq. (3); $\mathcal{E}_{B d l}$ is obtained using each data point's $\int B d l$ in Eq. (4) or (5). The dashed and solid lines show the ratios predicted by [26-28] and [29,30], respectively. Note that points (a) and (b) overlap, as do points (e)-(h). The data for all points are listed in Table I.

TABLE I. Data for Figs. 3 and 7-9 along with relevant experimental parameters for this experiment and earlier experiments [31]. The letters (a)-(p) in column 1 stand for the references listed in [31], while the letters $p, d$, and $e$ in column 3 denote protons, deuterons, and electrons, respectively. We assumed a $\pm 10 \%$ error in the rf magnets' $\int B d l$ for the experiments at IUCF and MIT, and a $\pm 5 \%$ error in the rf dipole's $\int B d l$ for our experiments at COSY. Note that, for the bottom 12 COSY proton points, $\nu_{y}$ was varied as explained in Figs. 5 and 6.

\begin{tabular}{|c|c|c|c|c|c|c|c|c|c|c|c|c|c|}
\hline & Ring & & $\begin{array}{l}\text { Magnet } \\
\text { type }\end{array}$ & $\begin{array}{c}f_{c} \\
(\mathrm{MHz})\end{array}$ & $\begin{array}{c}p \\
(\mathrm{GeV} / c)\end{array}$ & $\begin{array}{l}\int B_{\mathrm{rms}} d l \\
(\mathrm{~T} \mathrm{~mm})\end{array}$ & $\begin{array}{c}\mathcal{E}_{B d l} \\
\left(\times 10^{-6}\right)\end{array}$ & Flips & $\begin{array}{c}\Delta t(P=0) \\
(\mathrm{ms})\end{array}$ & $\begin{array}{c}\Delta f \\
(\mathrm{kHz})\end{array}$ & $\Gamma \equiv \frac{\Delta f / \Delta t}{2 \pi f_{c}{ }^{2}}$ & $\begin{array}{c}\mathcal{E}_{F S} \\
\left(\times 10^{-6}\right)\end{array}$ & $\begin{array}{l}\mathcal{E}_{F S} / \mathcal{E}_{B d l} \\
\left(\times 10^{-6}\right)\end{array}$ \\
\hline $\mathrm{a}$ & COSY & $p$ & Dipole & 1.47117 & 1.941 & 0.11 & 19.59 & 11 & 849 & 10 & $8.66 \times 10^{-10}$ & $19.7 \pm 0.3$ & $1.00 \pm 0.05$ \\
\hline $\mathrm{b}$ & & & & & & & & 1 & 750 & 10 & $9.81 \times 10^{-10}$ & $20.7 \pm 0.2$ & $1.06 \pm 0.05$ \\
\hline $\mathrm{c}$ & COSY & $p$ & Dipole & 1.49189 & 2.100 & 0.46 & 79.80 & 11 & 0.541 & 12 & $1.59 \times 10^{-6}$ & $840 \pm 30$ & $10.5 \pm 0.6$ \\
\hline $\mathrm{d}$ & & & & & & & & 1 & 0.753 & 8 & $7.60 \times 10^{-7}$ & $583 \pm 9$ & $7.3 \pm 0.4$ \\
\hline $\mathrm{e}$ & IUCF & $p$ & Dipole & 1.59784 & 0.489 & 0.16 & 66.70 & 1 & 6.97 & 10 & $8.94 \times 10^{-8}$ & $199 \pm 2$ & $3.0 \pm 0.3$ \\
\hline $\mathrm{f}$ & & & & & & & & 10 & 6.77 & 10 & $9.21 \times 10^{-8}$ & $202 \pm 4$ & $3.0 \pm 0.3$ \\
\hline $\mathrm{g}$ & IUCF & $p$ & Dipole & 1.59784 & 0.489 & 0.17 & 70.87 & 10 & 6.72 & 10 & $9.28 \times 10^{-8}$ & $204 \pm 3$ & $2.9 \pm 0.3$ \\
\hline $\mathrm{h}$ & & & & & & & & 1 & 6.56 & 10 & $9.50 \times 10^{-8}$ & $210 \pm 9$ & $3.0 \pm 0.3$ \\
\hline i & IUCF & $p$ & Dipole & 1.9658 & 0.649 & 0.042 & 14.03 & 1 & 4.03 & 4 & $4.09 \times 10^{-8}$ & $150 \pm 40$ & $11 \pm 3$ \\
\hline $\mathrm{j}$ & IUCF & $p$ & Dipole & 1.59784 & 0.489 & 0.042 & 17.69 & 1 & 60.7 & 10 & $1.03 \times 10^{-8}$ & $71 \pm 3$ & $4.0 \pm 0.4$ \\
\hline $\mathrm{k}$ & IUCF & $p$ & Solenoid & 1.50490 & 0.454 & 1.131 & 469.9 & 1 & 9.19 & 2 & $1.53 \times 10^{-8}$ & $95 \pm 8$ & $0.20 \pm 0.03$ \\
\hline 1 & IUCF & $p$ & Solenoid & 1.6973 & 0.529 & 0.99 & 352.7 & 1 & 4.86 & 3.5 & $3.98 \times 10^{-8}$ & $133 \pm 2$ & $0.38 \pm 0.04$ \\
\hline $\mathrm{m}$ & $\operatorname{COSY}$ & $d$ & Dipole & 1.14743 & 1.850 & 0.54 & 15.75 & 1 & $7.81 \times 10^{3}$ & 0.2 & $3.10 \times 10^{-12}$ & $1.166 \pm 0.009$ & $0.074 \pm 0.004$ \\
\hline $\mathrm{n}$ & COSY & $d$ & Dipole & 1.14743 & 1.850 & 0.15 & 4.375 & 1 & $9.76 \times 10^{4}$ & 0.1 & $1.24 \times 10^{-13}$ & $0.298 \pm 0.006$ & $0.068 \pm 0.004$ \\
\hline o & IUCF & $d$ & Solenoid & 1.67755 & 1.042 & 0.70 & 38.88 & 1 & 356 & 4 & $6.35 \times 10^{-10}$ & $17.3 \pm 0.6$ & $0.44 \pm 0.05$ \\
\hline $\mathrm{p}$ & MIT & $e$ & Dipole & 1.57616 & 0.670 & 0.070 & 17.78 & 1 & $2.07 \times 10^{3}$ & 2 & $6.18 \times 10^{-11}$ & $5.6 \pm 0.4$ & $0.32 \pm 0.04$ \\
\hline \multicolumn{14}{|c|}{ This experiment } \\
\hline & COSY & $d$ & Dipole & 1.14743 & 1.850 & 0.60 & 17.50 & 1 & $8.81 \times 10^{3}$ & 0.2 & $2.74 \times 10^{-12}$ & $1.101 \pm 0.006$ & $0.063 \pm 0.002$ \\
\hline & COSY & $p$ & Dipole & 1.49189 & 2.100 & 0.57 & 99.14 & 1 & 0.628 & 8 & $9.11 \times 10^{-7}$ & $634 \pm 10$ & $6.4 \pm 0.3$ \\
\hline & & & & & & 0.20 & 35.02 & 1 & 1.34 & 8 & $4.27 \times 10^{-7}$ & $434 \pm 2$ & $12.4 \pm 0.6$ \\
\hline & & & & & & 0.20 & 35.02 & 1 & 0.508 & 8 & $1.13 \times 10^{-6}$ & $705 \pm 3$ & $20 \pm 1$ \\
\hline & & & & & & 0.20 & 35.02 & 1 & 0.259 & 8 & $2.21 \times 10^{-6}$ & $988 \pm 9$ & $28.2 \pm 1.4$ \\
\hline & & & & & & 0.013 & 2.262 & 1 & 6.62 & 8 & $8.64 \times 10^{-8}$ & $195 \pm 1$ & $86 \pm 4$ \\
\hline & & & & & & 0.013 & 2.262 & 1 & 46.6 & 8 & $1.23 \times 10^{-8}$ & $73.6 \pm 0.3$ & $32.6 \pm 1.6$ \\
\hline & & & & & & 0.013 & 2.262 & 1 & 124 & 8 & $4.62 \times 10^{-9}$ & $45.2 \pm 0.2$ & $20 \pm 1$ \\
\hline & & & & & & 0.025 & 4.359 & 1 & 99.7 & 8 & $5.74 \times 10^{-9}$ & $50.3 \pm 0.2$ & $11.5 \pm 0.6$ \\
\hline & & & & & & 0.025 & 4.359 & 1 & 204 & 8 & $2.80 \times 10^{-9}$ & $35.2 \pm 0.5$ & $8.1 \pm 0.4$ \\
\hline & & & & & & 0.098 & 16.93 & 1 & 14.0 & 8 & $4.10 \times 10^{-8}$ & $135 \pm 1$ & $7.9 \pm 0.4$ \\
\hline & & & & & & 0.045 & 7.820 & 1 & 98.6 & 8 & $5.80 \times 10^{-9}$ & $50.6 \pm 0.4$ & $6.5 \pm 0.3$ \\
\hline & & & & & & 0.078 & 13.48 & 1 & 37.7 & 8 & $1.52 \times 10^{-8}$ & $81.9 \pm 0.3$ & $6.07 \pm 0.04$ \\
\hline
\end{tabular}


Fig. 5. There were no intrinsic spin resonances for deuterons near the operating tunes; thus, this deviation can not be due to a nearby intrinsic spin resonance.

In an effort to experimentally determine what might be causing these huge deviations in $\mathcal{E}_{F S} / \mathcal{E}_{B d l}$, we plotted the resonance strength ratios $\mathcal{E}_{F S} / \mathcal{E}_{B d l}$, including the new experimental data, in Figs. 7-9 against three different parameters:

Fig. 7: ramp time $\Delta t$ where $P=0$ in each $\Delta t$ curve,

Fig. 8: crossing rate $\Gamma$ where $P=0$ in each $\Delta t$ curve,

Fig. 9: frequency ramp range $\Delta f$ in each $\Delta t$ curve. The data seem scattered randomly in both Figs. 7 and 8 . There is much less scatter in Fig. 9: all data with small $\Delta f$ have $\mathcal{E}_{F S} / \mathcal{E}_{B d l}$ values far below 1, while all data with large $\Delta f$ have $\mathcal{E}_{F S} / \mathcal{E}_{B d l}$ values near or above 1 .

Clearly there still is no complete understanding of what is causing these very large resonance strength deviations. Figure 6 indicates that the large deviations above the predicted values of $1[26-28]$ and $0.5[29,30]$ are due to a nearby intrinsic spin resonance. Figure 9 suggests that the deviations below these lines could be due to the small $\Delta f$ frequency ramp range. Figure 9 also shows that, for deuterons, all $\mathcal{E}_{F S} / \mathcal{E}_{B d l}$ ratios are far below 1 for rf dipoles, but closer to 1 for rf solenoids. This may suggest anomalous behavior of spin-1 deuterons when spin-manipulated by dipoles.

Studying these deviations helped inspire a theoretical paper [45], which may be the first generalization of the Froissart-Stora equation [9] since its publication in 1960.

\section{SUMMARY AND CONCLUSION}

In summary, by compiling existing data and fitting it to the Froissart-Stora equation, we first found 10-20-fold deviations from the spin resonance strength equation that has been used for many years. We recently studied these deviations experimentally at COSY. We found no dependence on the beam's vertical size; however, we did find even larger deviations, of almost 100, when the rf spin resonance was near an intrinsic spin resonance. This proximity to an intrinsic resonance probably explains the 100times-larger-than-predicted deviations. The 10-20-timessmaller-than-predicted deviations for deuterons could be due to the following: the small $\Delta f$ sweep used to flip the deuteron spin; the resulting very slow crossing rate (Fig. 8); or some unexpected behavior of spin-1 deuterons in an rf dipole. We plan to test these and other possibilities at COSY, to learn if any are valid.

By adiabatically ramping the frequency of an rf dipole or rf solenoid through an rf-induced spin resonance, one can flip the polarization of a stored beam of protons, electrons or deuterons with an efficiency of up to $99.9 \%$. An rf dipole only slightly stronger than our $0.5 \mathrm{Tmm}$ dipole should allow efficient spin flipping of polarized protons in the $200 \mathrm{GeV}$ RHIC and perhaps someday in the $7 \mathrm{TeV}$ LHC.
Thus, these sometimes 100 -fold deviations must be better understood both theoretically and experimentally.

\section{ACKNOWLEDGMENTS}

We thank the IUCF, MIT and COSY staffs for the successful operation of their facilities. We also thank B. B. Blinov, A. W. Chao, E. D. Courant, Ya. S. Derbenev, D. Eversheim, H. Huang, W. Lorenzon, H. Rohdjeß, T. Roser, H. Sato, W. Scobel, K. Yonehara and others for their earlier help and advice. This research was supported by grants from the German BMBF Science Ministry.

[1] B. v. Przewoski et al., Phys. Rev. C 58, 1897 (1998).

[2] P. K. A. De Witt Huberts, Nucl. Phys. A 553, 845C (1993).

[3] R. Alarcon et al. (BLAST Collaboration), in MIT Bates Report No. 2-39, 1999.

[4] H. Rohdjess et al., in SPIN 2002: 15th International Spin Physics Symposium and Workshop on Polarized Electron Sources and Polarimeters, edited by Y. I. Makdisi, A. U. Luccio, and W.W. MacKay, AIP Conf. Proc. No. 675 (AIP, New York, 2003), p. 523.

[5] A. Assmann et al., in Proceedings of the Conference on High Energy Spin Physics, Bloomington, Indiana, 1994, edited by K. J. Heller and S. L. Smith, AIP Conf. Proc. No. 343 (AIP, New York, 1995), p. 219.

[6] Y. Makdisi, in Proceedings of the Conference on High Energy Spin Physics, Bloomington, Indiana, 1994 (Ref. [5]), p. 75.

[7] A.D. Krisch et al. (SPIN Collaboration), University of Michigan Report No. UM-HE 96-20, 1996; University of Michigan Report No. UM-HE 99-05, 1999.

[8] A. Airapetian et al. (HERMES Collaboration), DESYPRC Report No. 99-08, 1999.

[9] M. Froissart and R. Stora, Nucl. Instrum. Methods 7, 297 (1960).

[10] E. D. Courant, Bull. Am. Phys. Soc. 7, 33 (1962); Report No. BNL-EDC-45, 1962.

[11] B. W. Montague, Phys. Rep. 113, 1 (1984), Sec. 4.4.1.

[12] D. D. Caussyn et al., Phys. Rev. Lett. 73, 2857 (1994).

[13] D. A. Crandell et al., Phys. Rev. Lett. 77, 1763 (1996).

[14] B. von Przewoski et al., Rev. Sci. Instrum. 67, 165 (1996).

[15] B. B. Blinov et al., Phys. Rev. Lett. 81, 2906 (1998); V. A. Anferov et al., in Proceedings of the 13th International High Energy Spin Physics Symposium, Protvino, 1998, edited by N.E. Tyurin et al. (World Scientific, Singapore, 1999), p. 503.

[16] V.A. Anferov et al., Phys. Rev. ST Accel. Beams 3, 041001 (2000).

[17] B. B. Blinov et al., Phys. Rev. ST Accel. Beams 3, 104001 (2000).

[18] A. M. T. Lin et al., in SPIN 2000: 14th International Spin Physics Symposium, AIP Conf. Proc. No. 570 (AIP, New York, 2001), p. 736.

[19] V.S. Morozov et al., Phys. Rev. ST Accel. Beams 4, 104002 (2001).

[20] B. B. Blinov et al., Phys. Rev. Lett. 88, 014801 (2002); V.S. Morozov et al., in SPIN 2002: 15th International 
Spin Physics Symposium and Workshop on Polarized Electron Sources and Polarimeters (Ref. [4]), p. 776.

[21] V. S. Morozov et al., Phys. Rev. Lett. 91, 214801 (2003).

[22] K. Yonehara et al., in Intersections of Particle and Nuclear Physics: 8th Conference; CIPANP2003, edited by Z. Parsa, AIP Conf. Proc. No. 698 (AIP, New York, 2003), p. 763.

[23] M. A. Leonova et al., Phys. Rev. Lett. 93, 224801 (2004).

[24] V.S. Morozov et al., Phys. Rev. ST Accel. Beams 7, 024002 (2004).

[25] V.S. Morozov et al., Phys. Rev. ST Accel. Beams 8, 061001 (2005).

[26] S. Y. Lee, Spin Dynamics and Snakes in Synchrotrons (World Scientific, Singapore, 1997), p. 79, Eq. (4.85).

[27] H. Stockhorst and B. Lorentz, COSY internal report, 2003 (unpublished).

[28] V.S. Morozov et al., Phys. Rev. ST Accel. Beams 8, 099002 (2005).

[29] T. Roser, Handbook of Accelerator Physics and Engineering, edited by A. Chao and M. Tigner (World Scientific, Singapore, 2002), p. 153, Eq. (7).

[30] M. Bai et al., Phys. Rev. ST Accel. Beams 8, 099001 (2005).

[31] (a) V. S. Morozov et al., Phys. Rev. ST Accel. Beams 7, 024002 (2004); (b) 7, 024002 (2004); (c) M. A. Leonova et al., Phys. Rev. Lett. 93, 224801 (2004); (d) 93, 224801 (2004); (e) B. B. Blinov et al., Phys. Rev. Lett. 88, 014801 (2002); (f) 88, 014801 (2002); (g) Run SISN, 2001 (unpublished); (h) Run SISN, 2001 (unpublished); (i) V. A. Anferov et al., Phys. Rev. ST Accel. Beams 3, 041001 (2000); (j) B. B. Blinov et al., Phys. Rev. ST Accel. Beams 3, 104001 (2000); (k) B. B. Blinov et al., Phys. Rev. Lett. 81, 2906 (1998); (1) D. D. Caussyn et al., Phys. Rev. Lett. 73, 2857 (1994); (m) V. S. Morozov et al., Phys. Rev. ST Accel. Beams 8, 061001 (2005); (n) K. Yonehara et al., in Intersections of Particle and Nuclear Physics: 8th Conference; CIPANP2003 (Ref. [22]), p. 763; (o) V.S.
Morozov et al., Phys. Rev. Lett. 91, 214801 (2003); (p) V.S. Morozov et al., Phys. Rev. ST Accel. Beams 4, 104002 (2001).

[32] R. Maier, Nucl. Instrum. Methods Phys. Res., Sect. A 390, 1 (1997).

[33] A. Lehrach et al., in Proceedings of the Particle Accelerator Conference, New York, 1999, edited by A. Luccio and W. MacKay (IEEE, Piscataway, NJ, 1999), p. 2292.

[34] H. Stockhorst et al., in Proceedings of the 8th European Particle Accelerator Conference, Paris, 2002 (EPS-IGA/ CERN, Geneva, 2002), p. 629.

[35] A. Lehrach et al., in SPIN 2002: 15th International Spin Physics Symposium and Workshop on Polarized Electron Sources and Polarimeters (Ref. [4]), p. 153.

[36] V. Schwarz et al., in Proceedings of the 13th International High Energy Spin Physics Symposium, Protvino, 1998, edited by N.E. Tyurin (World Scientific, Singapore, 1999), p. 560.

[37] H. Stein et al., At. Energ. 94, 24 (2003).

[38] P.D. Eversheim et al., in Polarization Phenomena in Nuclear Physics: Eighth International Symposium, edited by E. J. Stephenson and S. E. Vigdor, AIP Conf. Proc. No. 339 (AIP, New York, 1995), p. 668.

[39] R. Weidmann et al., Rev. Sci. Instrum. 67, 1357 (1996).

[40] O. Felden et al., in Proceedings of the 9th International Workshop on Polarized Sources and Targets, Nashville, IN, 2001, edited by V. P. Derenchuk and B. von Przewoski (World Scientific, Singapore, 2002), p. 200.

[41] J. E. Goodwin et al., Phys. Rev. Lett. 64, 2779 (1990).

[42] V. A. Anferov et al., Phys. Rev. A 46, R7383 (1992).

[43] M. Bai et al., Phys. Rev. Lett. 80, 4673 (1998), Eq. (4).

[44] S. Y. Lee, Spin Dynamics and Snakes in Synchrotrons (World Scientific, Singapore, 1997), p. 27, Eq. (3.4).

[45] A. W. Chao, Phys. Rev. ST Accel. Beams 8, 104001 (2005). 